\title{
Disentangling the subcycle electron momentum spectrum in strong-field ionization
}

\author{
Nicholas Werby, ${ }^{1,2}$ Adi Natan $\odot,{ }^{1,2}$ Ruaridh Forbes, ${ }^{1,2}$ and Philip H. Bucksbaum ${ }^{1,2,3}$ \\ ${ }^{1}$ Stanford PULSE Institute, SLAC National Accelerator Laboratory, 2575 Sand Hill Road, Menlo Park, California 94025, USA \\ ${ }^{2}$ Department of Physics, Stanford University, Stanford, California 94305, USA \\ ${ }^{3}$ Department of Applied Physics, Stanford University, Stanford, California 94305, USA
}

(Received 18 February 2021; accepted 1 April 2021; published 22 April 2021)

\begin{abstract}
Quantum calculations of tunneling in strong-field ionization predict intricate momentum distributions due to sub-laser-cycle attosecond electron dynamics. These are obscured in most experiments by the dominance of intercycle interference patterns, which are the hallmark of above-threshold ionization (ATI). Highly controlled one- to two-cycle laser pulses produce less intercycle interference but cannot accurately recreate the subcycle features produced by uniform-cycle calculations due to the effect of the carrier envelope of the pulse. We present a simple and effective technique to recover these subcycle features in experimental multicycle spectra. We timefilter the momentum distribution to highlight features originating from the interference of electron trajectory pairs with ionization times less than one field cycle apart. This method removes the ATI patterns and reveals subcycle interference structures in detail. We can resolve modulations in holographic structures that provide a reference for comparing with calculations.
\end{abstract}

DOI: 10.1103/PhysRevResearch.3.023065

\section{INTRODUCTION}

When an atom or molecule is photoionized by a strong laser field, the photoelectron undergoes a series of complex field-driven dynamics [1-10]. Electron vector momentum distributions obtained using angle-resolving techniques, such as velocity map imaging (VMI), contain detailed information on rapidly evolving molecular geometries of the parent [11-13], holographic structures from the interference of electron trajectories [4-7,10,14-18], and nonlinear electron interactions with the strong field [19]. Each of these processes can produce patterns in the spectrum with characteristic features; however, patterns from different processes usually overlap on the electron detector, which impedes straightforward analysis. For experiments in the strong-field ionization (SFI) regime, where the ionization during each field cycle is significant, either running parameters such as ellipticity or field shape must be carefully chosen to emphasize specific dynamics, or significant analysis must be performed on the observed spectrum to disentangle these patterns to make the desired measurement.

A primary way to isolate individual features is to compare the measured spectrum with quantum SFI calculations. In the strong-field regime, electron spectra are largely determined by the phase of the laser field at the moment of photoionization, and the subsequent evolution in the field. This is known as the strong-field approximation (SFA) [20,21]. Of particular

Published by the American Physical Society under the terms of the Creative Commons Attribution 4.0 International license. Further distribution of this work must maintain attribution to the author(s) and the published article's title, journal citation, and DOI. interest for the characterization of the atomic or molecular target are interference patterns produced by distinct electron trajectory pairs ending up at the same final momentum whose ionization times are within a single laser field cycle of each other. These features are labeled as subcycle, whereas features produced by electron trajectory pairs ionized at least one full field cycle apart we label as intercycle. Referring to Fig. 1(d), electron trajectories resulting from different field cycles are roughly periodic and change very little between field cycles. Because interferences due to intercycle trajectory pairs are dependent primarily on the shape of the laser pulse rather than any property of the target, they do not encode any new information about the target, but they still produce additional undesired features in the momentum distribution [15]. Importantly, angle-resolved SFI spectra produced by a multicycle laser pulse contain structures produced from both subcycle and intercycle interfering trajectory pairs. To mitigate this, calculations of subcycle features often assume strong-field conditions that are practically unattainable, such as a few uniform strong-field cycles that turn on and off instantly $[15,16]$. Experiments cannot mimic this. Instead, realizable ultrashort one- or two-cycle laser pulses include a time-varying field envelope, which gives rise to a carrier envelope phase (CEP) parameter that governs the electron dynamics but is absent from calculations that assume uniform cycles (see Fig. 1) $[22,23]$. These pulses generate spectra that do not match subcycle calculations, despite having eliminated intercycle features. Thus to complement the ultrashort approach and more closely produce experimental spectra comparable to these few-cycle calculations, we require a different approach.

We are interested in subcycle interfering electron trajectory pairs in SFI generated by a standard 30-50-fs multicycle laser pulse from a commercial Ti:sapphire laser system. This requires identifying and distinguishing the key features of 


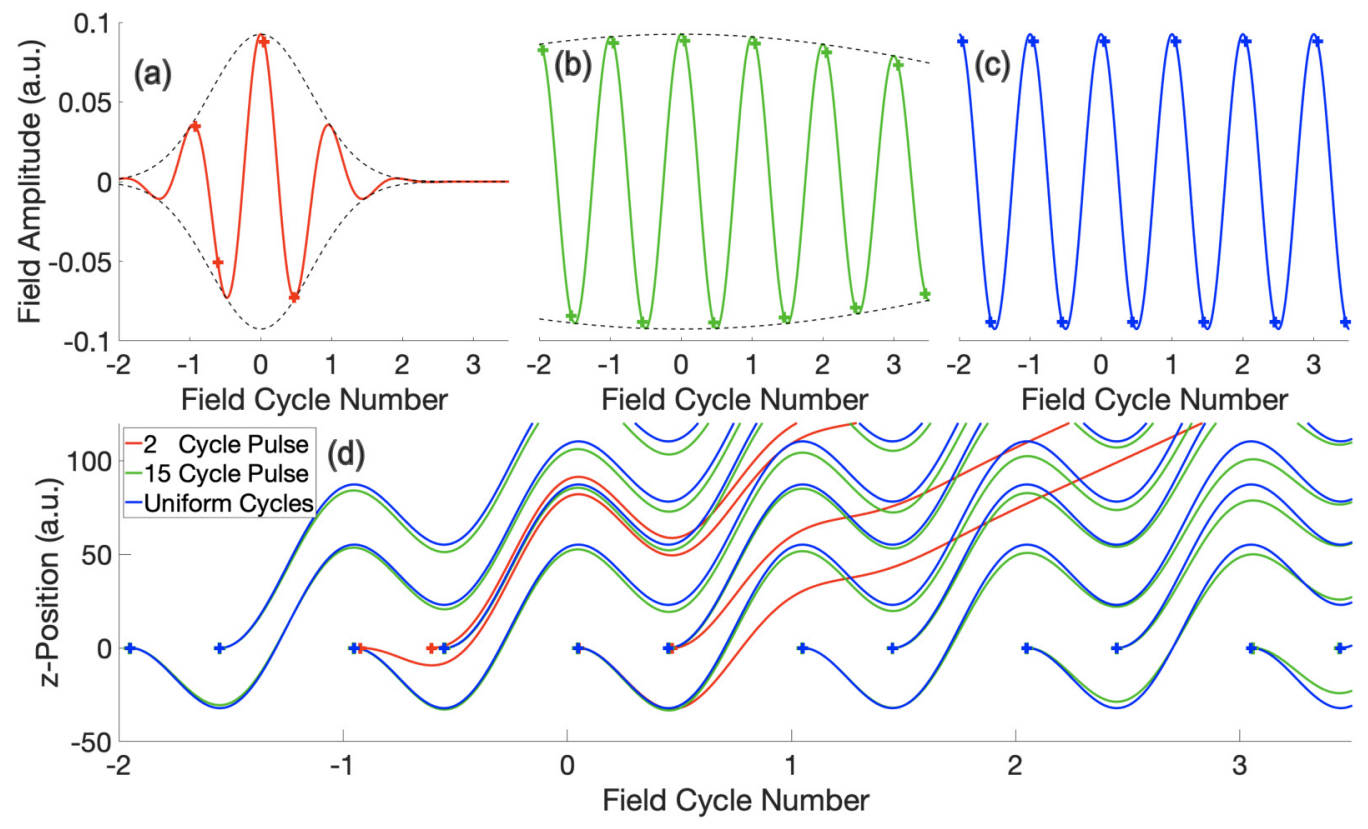

FIG. 1. Comparison of the photoelectron trajectories produced by three distinct 800-nm laser pulses. (a) and (b) show the electric field of a 2 -cycle $(\sim 5.3 \mathrm{fs})$ pulse and a 15-cycle $(\sim 40 \mathrm{fs})$ pulse, respectively. The carrier envelope is indicated by the dashed black lines for each. (c) shows a uniform-cycle laser field pulse. In (a)-(c) the ionization times are plotted as crosses for an electron which ends up at $\left(p_{\|}, p_{\perp}\right)=(0.5,0)$ on the detector (see text). (d) displays the electron trajectories propagating in each of the laser fields above. Each trajectory begins at the time of ionization indicated in (a), (b), or (c) and arrives at the same final momentum (0.5, 0). The 15-cycle pulse much better reproduces the trajectories generated by the uniform-cycle pulse, as the carrier envelope parameter has less of an impact. For simplicity, no rescattering trajectories are shown.

intercycle dynamics and subcycle dynamics present in multicycle spectra. In the tunneling ionization regime, the primary intercycle features appear as a comb of above-threshold ionization (ATI) peaks, which are equally spaced by the energy of the driving laser photons $[24,25]$. Under ordinary conditions, this comb dominates the momentum spectrum and frequently obscures patterns generated by subcycle dynamics. This is most noticeable in the direct ionization regime of the spectrum below $2 U_{\mathrm{p}}$, where $U_{\mathrm{p}}$ is the ponderomotive energy of a free electron in the laser field [26]. Here, holographic features resulting from the interference of electron trajectories after ionization are prevalent $[14,15]$. These subcycle features would be far more visible if the ATI contribution to the spectrum could be filtered out.

In this paper, we present a time-filtering technique that effectively eliminates interference patterns from trajectory pairs ionized at least one field cycle apart, which removes the energy-periodic background ATI comb in multicycle photoelectron momentum spectra. This suppresses the intercycle contributions to the spectrum, thus leaving only the subcycle dynamics. Importantly, since we use a multicycle laser pulse, the resultant spectrum closely resembles that due to a single cycle of a steady-state laser field [see Figs. 1(b)1(d)], which models common SFI calculations more closely $[2,15,16,27-30]$. Furthermore, the time-filtering technique is broadly applicable to angle-resolved SFI electron spectra and is independent of experimental parameters, making it a simple tool for extracting subcycle structures from multicycle data. To illustrate the time filtering, we demonstrate it on a high-fidelity VMI spectrum of argon gas photoionized with an approximately $40-\mathrm{fs}, 800-\mathrm{nm}$ commercial Ti:sapphire laser at a peak intensity of $200 \mathrm{TW} / \mathrm{cm}^{2}$. This time-filtering method reveals features of subcycle ionization that should be of interest in the study of holography in SFI. All quantities are in atomic units, unless otherwise stated.

\section{EXTRACTING THE SUBCYCLE SPECTRUM}

The raw VMI detector image requires several layers of processing to reach the effective single-cycle spectrum. The steps are outlined below and in Fig. 2. The two-dimensional VMI raw detector image is first calibrated to measure the transverse momentum of the electrons striking it [31]. The resulting image is a projection on $\left(p_{\|}, p_{\perp}\right)$ of a three-dimensional Newton sphere of electron momenta $\left(p_{\|}, p_{\perp}, \phi\right)$ [32]. Here, $p_{\|}$is the momentum magnitude parallel to the polarization axis of the ionizing field, $p_{\perp}$ is the momentum magnitude transverse to the polarization axis, and $\phi$ is the azimuthal angle about the the polarization axis. Since photoionization with linearly polarized light is cylindrically symmetric about the polarization $(\|)$ axis, reconstructing just the $p_{\|}-p_{\perp}$ slice of the Newton sphere from its full projection is sufficient to display the full three-dimensional information [33].

Reconstructing this momentum slice begins by symmetrizing the VMI data. Each raw spectrum is centered and rotated, and four-quadrant symmetry is imposed by averaging each pixel in the raw image with its counterparts in the other three quadrants.

The projection is inverted through the procedure of polar onion peeling [34]. Beginning from the outermost radius of the raw data, we construct the Legendre decomposition leastsquares fit to the photoelectron angular distribution (PAD) 


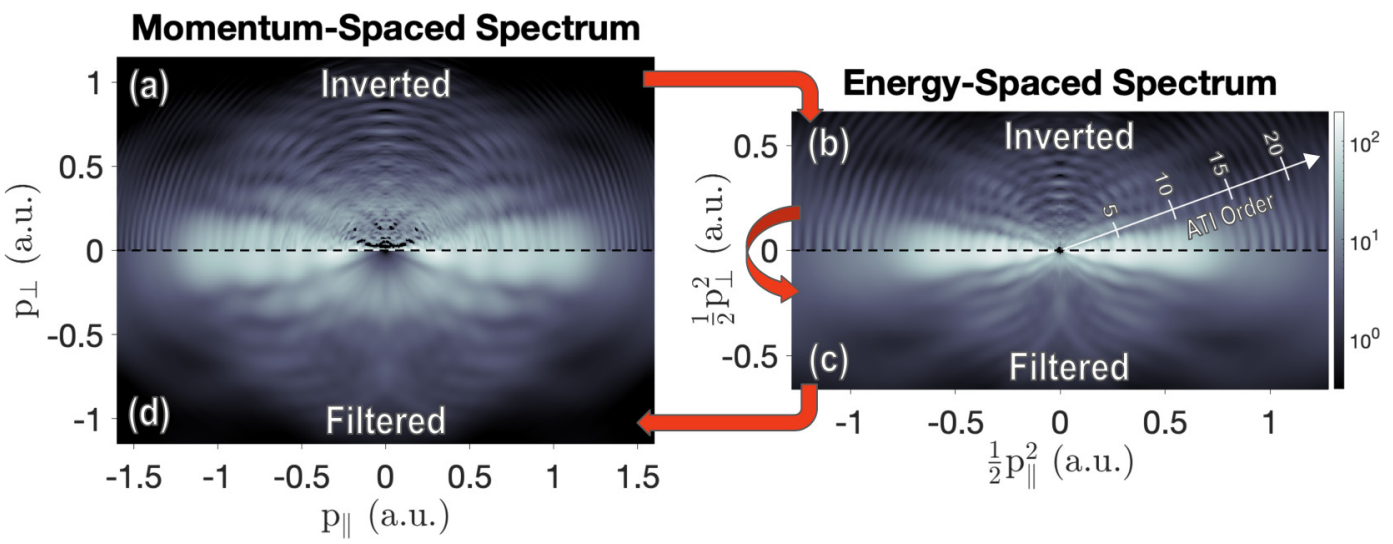

FIG. 2. The application of the time-filtering technique to our argon spectrum. Here, we have zoomed into the spectrum to emphasize the region within $2 U_{\mathrm{p}}$ where the filtering has the greatest effect. The red arrows outline the order of the data processing, and the color scale indicates the normalized photoelectron yield. (a) The top half of the image shows the reconstructed $p_{\|}-p_{\perp}$ momentum slice from polar onion peeling [34]. (b) Transforming the onion-peeled spectrum to a polar energy plot yields the top half of the image. In this representation the ATI rings are equally spaced, the radial axis counts the ATI order, and the $x$ and $y$ axes label the projection of the squared components of momenta as indicated. (c) The bottom half shows the filtered energy-spaced spectrum as detailed in the text on the same scale. (d) The bottom half shows the filtered momentum slice on the same scale as the original spectrum. Importantly, for (c) and (d) we note that the time filtering has only removed the ATI rings, and all other visible structures remain unaffected.

at that radius using the Legendre basis polynomials of even orders $P_{n}[\cos (\theta)]$.

$$
I\left(\theta, p_{r}\right)=C_{0}\left(p_{r}\right) \sum_{n_{\text {even }}} \beta_{n}\left(p_{r}\right) P_{n}[\cos (\theta)] .
$$

Here, $\theta$ is the polar angle about the center of the twodimensional slice, and $I\left(\theta, p_{r}\right)$ is the PAD fit at the momentum radius $p_{r}$. The anisotropy parameters $\beta_{n}\left(p_{r}\right)$ are defined as $\beta_{n}\left(p_{r}\right)=\frac{C_{n}\left(p_{r}\right)}{C_{0}\left(p_{r}\right)}$, where $C_{n}\left(p_{r}\right)$ is the $n$th Legendre coefficient for the decomposition at $p_{r}$. In our decomposition, the maximum Legendre order is set to 42 , well beyond the required angular resolution for holographic predictions in SFI [15]. We then convolve this fit about the polarization axis and project the resulting spherical shell back to the image plane. This generates the projection contribution from that spherical shell of the Newton sphere. Subtracting this contribution from the spectrum "peels" this shell of the Newton sphere off the spectrum. Repeating this procedure for successively smaller radii yields the fully inverted spectrum [34-36].

This process generates the momentum slice $p_{\|}-p_{\perp}$ as a superposition of Legendre polynomials whose anisotropy parameters, $\beta_{n}\left(p_{r}\right)$, are one-dimensional functions of $p_{r}$. All of the information in the two-dimensional momentum distribution is now encoded in a one-dimensional $\beta_{n}\left(p_{r}\right)$ parameter for each included order $n$ [33].

At this point in the analysis the subcycle features are obscured by highly prominent ring structures of intercycle ATI interference [24]. The ATI appears as a comb of peaks in each order of $\beta_{n}\left(p_{r}\right)$ spaced by the photon energy of the ionizing laser, so we transform the spectral grid from momentum to energy by directly resampling $\beta_{n}\left(p_{r}\right) \rightarrow \beta_{n}\left(E_{r}\right)$. The result is shown in Fig. 2(b). Since in this spacing the peaks are periodic, we can take the Fourier transforms of each $\beta_{n}\left(E_{r}\right)$ [Fig. 3(b)] which contain a sharp peak due to the ATI. The reciprocal space of energy is time, and in this case the times are the time differences between two interfering electron trajectories, as highlighted in Fig. 1. We see a large spike at a time difference of one field cycle, which is consistent with the ATI forming due to the interference of similar trajectories from different laser cycles.

In this way we identify that subcycle interference patterns are encoded in $\tilde{\beta}_{n}(t)$ as all structures below the time difference of one field cycle. This suggests that in order to suppress the effect of intercycle interference features such as ATI rings we can Fourier-filter $\beta_{n}\left(E_{r}\right)$. We construct a zero-phase, finite impulse response low-pass filter, with a cutoff just below one field cycle to suppress the ATI peak and further intercycle interference features, without affecting any subcycle structures in the spectrum. A Kaiser window filter is selected to make the response of the signal maximally flat in the passband [see Fig. 3(c)]. The result of this filtering can be seen in Fig. 2(c). Transforming back to momentum spacing returns the original momentum grid, and the image can be reproduced according to Eq. (1). The final filtered spectrum does not contain the ATI interference pattern but does preserve the features that are caused by subcycle interference in field ionization and rescattering, as shown in Fig. 4.

Figure 4 resolves interference features in the holographic regime of the spectrum that are normally obscured by the ATI rings. Most strikingly, along the spider-leg structures, which have been studied extensively in the earlier literature [14,37-41], we identify subtle wiggling modulations. This is most clearly visible in the first-order spider leg in the range $0.4<p_{\|}<1$ and $0.15<p_{\perp}<0.4$, though the same modulations can be seen with increasing difficulty at higher-order legs. Additionally, we can resolve periodic minima along the polarization axis and each spider leg. Clear observations of these holographic features can help validate strong-field calculations and lead to a more complete understanding of the strong-field-driven dynamics that generate them. 

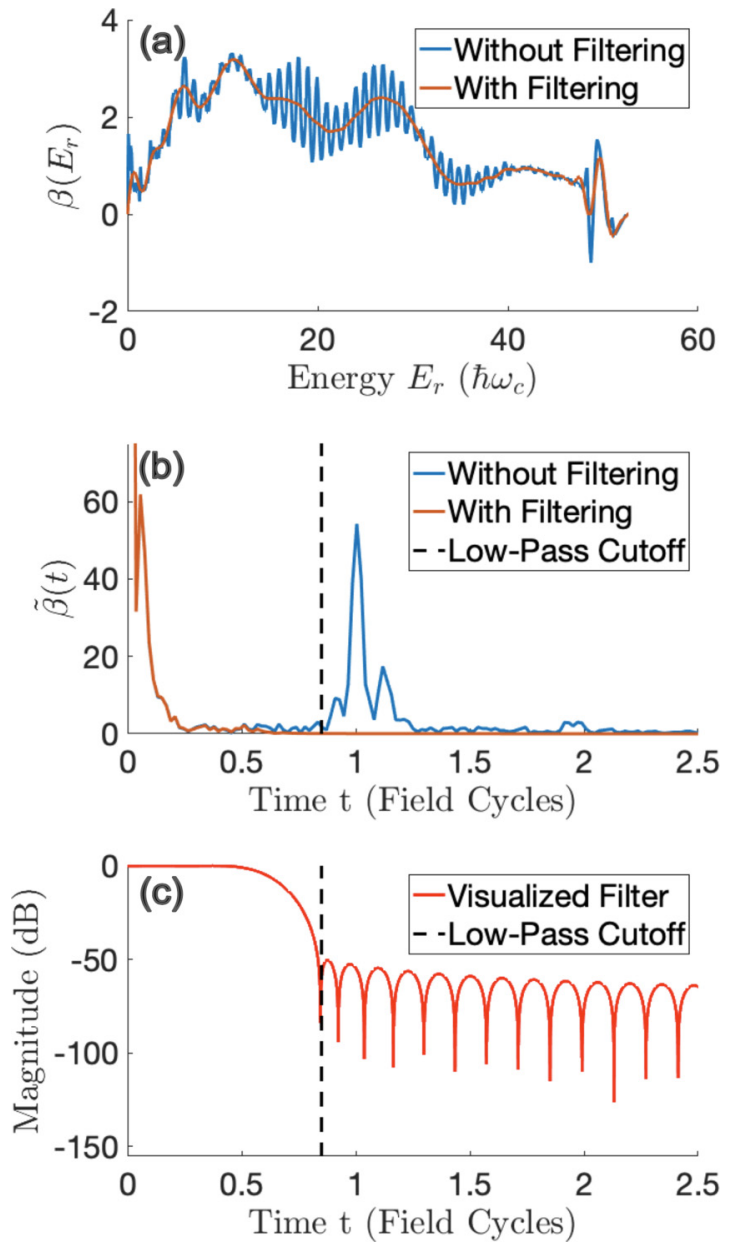

FIG. 3. (a) The $\beta_{6}\left(E_{r}\right)$ parameter of the spectrum in Fig. 2 presented in units of the energy of a single photon at the central laser frequency $\hbar \omega_{c}$. $\left[\beta_{6}\left(E_{r}\right)\right.$ is depicted as an example of the filtering method. The other orders are analyzed in an identical way.] Shown is the same parameter before (blue) and after (orange) time filtering. Prior to filtering we see the evenly spaced ATI comb quite prominently. After we apply the time filter we see that the filtering only removes the ATI comb and does not disrupt the underlying shape of the parameter. (b) The Fourier transform of $\beta_{6}\left(E_{r}\right)$. The large spike at $t=1$ field cycle is due to the ATI comb. A low-pass filter with a cutoff (black dashed line) just below this peak removes only the ATI comb. Below the cutoff, the filtered and unfiltered $\tilde{\beta}_{6}(t)$ match very closely. This is achieved by designing a filter that is maximally flat in the passband. (c) The magnitude response of the low-pass filter applied to each $\beta_{n}\left(E_{r}\right)$ parameter. Note the smoothness of the magnitude response in the passband below the cutoff, which helps prevent nonphysical artifacts from appearing in the data as a result of the filtering.

Although we have only demonstrated the time-filtering procedure using a single VMI spectrum, the idea behind it is quite general and broadly applicable to momentum-resolved electron spectra collected in other ways. The key takeaway is that intercycle interference patterns can be isolated and extracted through the Fourier transform of the yield of electrons versus the electron energy, which can be determined from the momentum spectrum. This Fourier transform describes the time difference between the ionization times of interfering

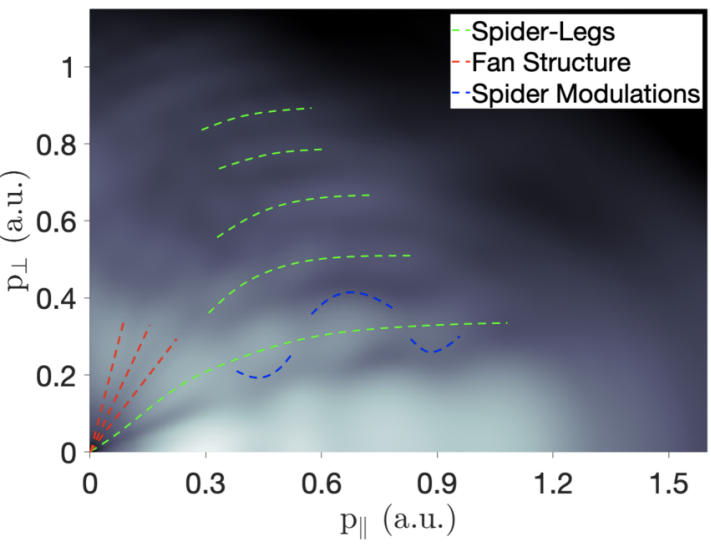

FIG. 4. Magnified image of a single quadrant of the filtered spectrum shown in Fig. 2(d) on the same logarithmic color scale. The spider-leg structure, fan structure, and wiggling modulations along the spider-leg structures are highlighted by dashed lines. Periodic minima along the polarization axis can also be seen. These modulations are normally obscured by the ATI rings.

electron trajectories, which should hold true for any method of momentum-resolved electron collection. Here, we outlined polar onion peeling as our inversion method, which uses the Legendre basis to construct the PAD fits before inverting to produce the $p_{\|}-p_{\perp}$ momentum slice. This too can be generalized. Polar onion peeling is advantageous for the time filtering as it produces one-dimensional, polar anisotropy parameters which can each be filtered individually to preserve the angular resolution of the spectrum. However, this can be achieved for any angularly resolved spectrum by constructing PAD fits after any desired inversion method and then filtering the produced coefficients as outlined previously in the text.

\section{CONCLUSION}

In conclusion, we have presented a procedure to filter out the ATI comb and other intercycle features in a strong-field ionization spectrum, to more clearly reveal the underlying subcycle features of the strong-field ionization process. This time filtering is not simply an aesthetic change. The filtered momentum spectrum shown in Fig. 4 contains holographic features from subcycle trajectory interferences that are normally obscured by the ATI rings [15]. These subcycle interferences revealed by the filtering merit significant further study and should help to validate holographic models in strong-field ionization.

\section{ACKNOWLEDGMENTS}

We would like to thank J. Cryan for invaluable help in the laboratory in getting the experimental apparatus up and running. We are also grateful to A. Maxwell and C. Faria for their very illuminating discussions on the nature of subcycle trajectory interferences in strong-field ionization and how they display in photoelectron momentum distributions. This work is supported by the U.S. Department of Energy, Office of Science, Basic Energy Sciences (BES), Chemical Sciences, Geosciences, and Biosciences Division, AMOS Program. 
[1] T. Okino, Y. Furukawa, Y. Nabekawa, S. Miyabe, A. A. Eilanlou, E. J. Takahashi, K. Yamanouchi, and K. Midorikawa, Direct observation of an attosecond electron wave packet in a nitrogen molecule, Sci. Adv. 1, e1500356 (2015).

[2] O. Smirnova, S. Patchkovskii, Y. Mairesse, N. Dudovich, and M. Y. Ivanov, Strong-field control and spectroscopy of attosecond electron-hole dynamics in molecules, Proc. Natl. Acad. Sci. USA 106, 16556 (2009).

[3] J. Xu, Z. Chen, A.-T. Le, and C. D. Lin, Self-imaging of molecules from diffraction spectra by laser-induced rescattering electrons, Phys. Rev. A 82, 033403 (2010).

[4] B. Willenberg, J. Maurer, U. Keller, J. Daněk, M. Klaiber, N. Teeny, K. Z. Hatsagortsyan, and C. H. Keitel, Holographic interferences in strong-field ionization beyond the dipole approximation: The influence of the peak and focalvolume-averaged laser intensities, Phys. Rev. A 100, 033417 (2019).

[5] J. Tan, Y. Zhou, M. He, Q. Ke, J. Liang, Y. Li, M. Li, and P. Lu, Time-resolving tunneling ionization via strong-field photoelectron holography, Phys. Rev. A 99, 033402 (2019).

[6] M. He, Y. Li, Y. Zhou, M. Li, W. Cao, and P. Lu, Direct Visualization of Valence Electron Motion Using Strong-Field Photoelectron Holography, Phys. Rev. Lett. 120, 133204 (2018).

[7] S. G. Walt, N. Bhargava Ram, M. Atala, N. I. ShvetsovShilovski, A. von Conta, D. Baykusheva, M. Lein, and H. J. Wörner, Dynamics of valence-shell electrons and nuclei probed by strong-field holography and rescattering, Nat. Commun. 8, 15651 (2017).

[8] Y. Zhou, O. I. Tolstikhin, and T. Morishita, Near-Forward Rescattering Photoelectron Holography in Strong-Field Ionization: Extraction of the Phase of the Scattering Amplitude, Phys. Rev. Lett. 116, 173001 (2016).

[9] M. Meckel, A. Staudte, S. Patchkovskii, D. M. Villeneuve, P. B. Corkum, R. Dörner, and M. Spanner, Signatures of the continuum electron phase in molecular strong-field photoelectron holography, Nat. Phys. 10, 594 (2014).

[10] T. Marchenko, Y. Huismans, K. J. Schafer, and M. J. J. Vrakking, Criteria for the observation of strong-field photoelectron holography, Phys. Rev. A 84, 053427 (2011).

[11] D.-D. T. Vu, N.-L. T. Phan, V.-H. Hoang, and V.-H. Le, Dynamic molecular structure retrieval from low-energy laserinduced electron diffraction spectra, J. Phys. B: At., Mol. Opt. Phys. 50, 245101 (2017).

[12] F. Krečinić, P. Wopperer, B. Frusteri, F. Brauße, J.-G. Brisset, U. De Giovannini, A. Rubio, A. Rouzée, and M. J. J. Vrakking, Multiple-orbital effects in laser-induced electron diffraction of aligned molecules, Phys. Rev. A 98, 041401(R) (2018).

[13] T. Morishita, M. Okunishi, K. Shimada, G. Prümper, Z. Chen, S. Watanabe, K. Ueda, and C. D. Lin, Retrieval of experimental differential electron-ion elastic scattering cross sections from high-energy ATI spectra of rare gas atoms by infrared lasers, J. Phys. B: At., Mol. Opt. Phys. 42, 105205 (2009).

[14] Y. Huismans, A. Rouzée, A. Gijsbertsen, J. H. Jungmann, A. S. Smolkowska, P. S. W. M. Logman, F. Lépine, C. Cauchy, S. Zamith, T. Marchenko, J. M. Bakker, G. Berden, B. Redlich, A. F. G. van der Meer, H. G. Muller, W. Vermin, K. J. Schafer, M. Spanner, M. Y. Ivanov, O. Smirnova et al.,
Time-resolved holography with photoelectrons, Science 331, 61 (2011).

[15] C. Figueira de Morisson Faria and A. S. Maxwell, It is all about phases: ultrafast holographic photoelectron imaging, Rep. Prog. Phys. 83, 034401 (2020).

[16] A. S. Maxwell and C. Figueira de Morisson Faria, Coulombfree and Coulomb-distorted recolliding quantum orbits in photoelectron holography, J. Phys. B: At., Mol. Opt. Phys. 51, 124001 (2018).

[17] M. Haertelt, X.-B. Bian, M. Spanner, A. Staudte, and P. B. Corkum, Probing Molecular Dynamics by Laser-Induced Backscattering Holography, Phys. Rev. Lett. 116, 133001 (2016).

[18] G. Porat, G. Alon, S. Rozen, O. Pedatzur, M. Krüger, D. Azoury, A. Natan, G. Orenstein, B. D. Bruner, M. J. J. Vrakking, and N. Dudovich, Attosecond time-resolved photoelectron holography, Nat. Commun. 9, 2805 (2018).

[19] C. I. Blaga, F. Catoire, P. Colosimo, G. G. Paulus, H. G. Muller, P. Agostini, and L. F. DiMauro, Strong-field photoionization revisited, Nat. Phys. 5, 335 (2009).

[20] F. H. M. Faisal, Multiple absorption of laser photons by atoms, J. Phys. B: At. Mol. Phys. 6, L89 (1973).

[21] H. R. Reiss, Semiclassical electrodynamics of bound systems in intense fields, Phys. Rev. A 1, 803 (1970).

[22] T. Wittmann, B. Horvath, W. Helml, M. G. Schätzel, X. Gu, A. L. Cavalieri, G. G. Paulus, and R. Kienberger, Single-shot carrier-envelope phase measurement of few-cycle laser pulses, Nat. Phys. 5, 357 (2009).

[23] M. F. Kling, J. Rauschenberger, A. J. Verhoef, E. Hasović, T. Uphues, D. B. Milošević, H. G. Muller, and M. J. J. Vrakking, Imaging of carrier-envelope phase effects in above-threshold ionization with intense few-cycle laser fields, New J. Phys. 10, 025024 (2008).

[24] R. R. Freeman, P. H. Bucksbaum, H. Milchberg, S. Darack, D. Schumacher, and M. E. Geusic, Above-Threshold Ionization with Subpicosecond Laser Pulses, Phys. Rev. Lett. 59, 1092 (1987).

[25] T. Marchenko, H. G. Muller, K. J. Schafer, and M. J. J. Vrakking, Wavelength dependence of photoelectron spectra in above-threshold ionization, J. Phys. B: At., Mol. Opt. Phys. 43, 185001 (2010).

[26] P. H. Bucksbaum, R. R. Freeman, M. Bashkansky, and T. J. McIlrath, Role of the ponderomotive potential in above-threshold ionization, J. Opt. Soc. Am. B 4, 760 (1987).

[27] O. Smirnova, Y. Mairesse, S. Patchkovskii, N. Dudovich, D. Villeneuve, P. Corkum, and M. Y. Ivanov, High harmonic interferometry of multi-electron dynamics in molecules, Nature (London) 460, 972 (2009).

[28] M.-H. Yuan, A. D. Bandrauk, and X.-B. Bian, Exploring recollision of ultrafast electrons from photoelectron momentum distributions using single-cycle near-infrared laser pulses, Phys. Rev. A 103, 013108 (2021).

[29] E. Pisanty and M. Ivanov, Slalom in complex time: Emergence of low-energy structures in tunnel ionization via complex-time contours, Phys. Rev. A 93, 043408 (2016). 
[30] D. B. Milošević, G. G. Paulus, D. Bauer, and W. Becker, Abovethreshold ionization by few-cycle pulses, J. Phys. B: At., Mol. Opt. Phys. 39, R203 (2006).

[31] D. A. Dahl, SIMION 3D Version 6.0 User's Manual, Technical Report INEL-95/0403-Rev.4, Idaho National Engineering and Environmental Laboratory (1995) .

[32] A. T. J. B. Eppink and D. H. Parker, Velocity map imaging of ions and electrons using electrostatic lenses: Application in photoelectron and photofragment ion imaging of molecular oxygen, Rev. Sci. Instrum. 68, 3477 (1997).

[33] K. L. Reid, Photoelectron angular distributions, Annu. Rev. Phys. Chem. 54, 397 (2003).

[34] G. M. Roberts, J. L. Nixon, J. Lecointre, E. Wrede, and J. R. R. Verlet, Toward real-time charged-particle image reconstruction using polar onion-peeling, Rev. Sci. Instrum. 80, 053104 (2009).

[35] M. J. J. Vrakking, An iterative procedure for the inversion of two-dimensional ion/photoelectron imaging experiments, Rev. Sci. Instrum. 72, 4084 (2001).

[36] G. A. Garcia, L. Nahon, and I. Powis, Two-dimensional charged particle image inversion using a polar basis function expansion, Rev. Sci. Instrum. 75, 4989 (2004).

[37] D. D. Hickstein, P. Ranitovic, S. Witte, X.-M. Tong, Y. Huismans, P. Arpin, X. Zhou, K. E. Keister, C. W. Hogle, B.
Zhang, C. Ding, P. Johnsson, N. Toshima, M. J. J. Vrakking, M. M. Murnane, and H. C. Kapteyn, Direct Visualization of Laser-Driven Electron Multiple Scattering and Tunneling Distance in Strong-Field Ionization, Phys. Rev. Lett. 109, 073004 (2012).

[38] X. Gong, P. He, Q. Song, Q. Ji, K. Lin, W. Zhang, P. Lu, H. Pan, J. Ding, H. Zeng, F. He, and J. Wu, Pathway-resolved photoelectron emission in dissociative ionization of molecules, Optica 3, 643 (2016).

[39] M. Möller, F. Meyer, A. M. Sayler, G. G. Paulus, M. F. Kling, B. E. Schmidt, W. Becker, and D. B. Milošević, Off-axis lowenergy structures in above-threshold ionization, Phys. Rev. A 90, 023412 (2014).

[40] Q. Li, X.-M. Tong, T. Morishita, H. Wei, and C. D. Lin, Fine structures in the intensity dependence of excitation and ionization probabilities of hydrogen atoms in intense 800-nm laser pulses, Phys. Rev. A 89, 023421 (2014).

[41] P. A. Korneev, S. V. Popruzhenko, S. P. Goreslavski, T.-M. Yan, D. Bauer, W. Becker, M. Kübel, M. F. Kling, C. Rödel, M. Wünsche, and G. G. Paulus, Interference Carpets in Above-Threshold Ionization: From the Coulomb-Free to the Coulomb-Dominated Regime, Phys. Rev. Lett. 108, 223601 (2012). 